\title{
¿Qué sirve en la prevención de la violencia juvenil?
}

\author{
Rodrigo Guerrero, MD, PhD. (I)
}

\begin{abstract}
Guerrero R.
¿Qué sirve en la prevención de la violencia juvenil? Salud Publica Mex 2008;50 supl I:S86-S92.

\section{Resumen}

Entre los adolescentes, que representan $20.3 \%$,y los jóvenes, que constituyen $31.6 \%$ de la población total de las Américas, la violencia alcanza proporciones epidémicas y se convierte en un serio problema de salud pública en la región. El presente trabajo es el resultado de una revisión documental sobre las intervenciones que han demostrado éxito en la prevención de este problema entre los jóvenes. De las intervenciones efectivas destaca la concesión de tiempo específico de los adultos para interactuar con niños y adolescentes, así como la aplicación de programas enfocados en el desarrollo infantil temprano y las prácticas educativas adecuadas entre los padres. Esta última es una de las intervenciones de mayor beneficio en términos del costo para la reducción de los comportamientos de riesgo entre los jóvenes. Los esfuerzos para desarmar a la población civil en periodos y espacios críticos sobresale también por su eficacia.
\end{abstract}

Palabras clave:violencia; juventud; intervenciones; prevención; Colombia

\section{Guerrero R.}

Youth violence prevention, what works?

Salud Publica Mex 2008;50 suppl I:S86-S92.

\begin{abstract}
Violence is currently considered to be both an epidemic problem and a public health problem among youth and adolescents, who represent $31.6 \%$ and $20.3 \%$ of the overall population of the Americas region, respectively. This paper analyzes the scientific literature on violence prevention as it relates to successful interventions among these populations. Some of the most successful interventions are those related to active engagement of adults with children and adolescents and programs oriented toward early child development and adequate educational practices among parents. The latter is one of the most cost-effective violence prevention interventions for children and adolescents. Efforts to disarm civilian populations during critical periods have also been shown to be effective.
\end{abstract}

Key words: violence; youth; interventions; prevention
L a violencia se encuentra en el origen de la humanidad, desde los albores de la historia registrada. Caín mató a su hermano Abel con la quijada de un burro, según cuenta la Biblia. ${ }^{1}$ Las guerras y los genocidios se hallan en el decurso de la especie humana y sólo apenas en fechas recientes se ha cuestionado la inevitabilidad de la violencia y se ha delineado su prevención.

Considerar la violencia como un asunto de salud pública es un hecho "relativamente reciente", escribió Everett Koop, el Cirujano General de EUA en 1985. Él

(I) Centro de Investigaciones de Salud y Violencia, Universidad del Valle San Fernando. Cali, Colombia.

Fecha de recibido: 3 de agosto de 2007 - Fecha de aceptado: 2 I de enero de 2008

Solicitud de sobretiros: Dr. Rodrigo Guerrero. Centro de Investigaciones de Salud y Violencia. Facultad de Salud - Oficina II4,

Universidad del Valle San Fernando. Calle 4B No. 36-00, Dirección Postal 25360. Cali, Colombia.

Correo electrónico: guerrerr@yahoo.com, rodgue@cable.net.co 
mismo declaró en 1992 que la violencia era una emergencia de salud pública. ${ }^{3}$

Con posterioridad, en 1993, los ministros de salud de las Américas declararon que la prevención de la violencia era una prioridad de la salud pública ${ }^{4}$ y en 1996 la Organización Mundial de la Salud (OMS) aprobó una resolución similar. ${ }^{5}$

En 1997 se formalizó una alianza de instituciones interesadas en la prevención de la violencia, la Coalición Interamericana para la Prevención de la Violencia, integrada por instituciones como la Oficina Panamericana de la Salud, el Banco Interamericano de Desarrollo, el Banco Mundial, los Centers for Desease Control de Estados Unidos de América, la Organización de Estados Americanos, la UNESCO y la USAID. ${ }^{6}$

Hoy, la prevención de la violencia ocupa un lugar relevante, no sólo en la agenda política de los países de la región sino también en los primeros planos de los medios de comunicación.

\section{Violencia en las Américas}

Entre los adolescentes, que representan 20.3\%, y los jóvenes, que constituyen $31.6 \%$ de la población total de las Américas, ${ }^{7}$ la violencia alcanza proporciones epidémicas y es un serio problema de salud en varios países de la región.

Las tasas más elevadas de mortalidad por homicidio ocurren en hombres, con edades de 15 a 14 años. ${ }^{8}$ Sin embargo, se observan grandes fluctuaciones, desde 267 y 93 para Colombia y Puerto Rico hasta 93 y 21 en Ecuador y Trinidad y Tobago. ${ }^{9}$

La violencia es la principal causa de pérdida de años de vida potencial entre los adolescentes y jóvenes de la mayoría de los países de las Américas, con una pérdida promedio de 30 a 40 años por muerte. ${ }^{7}$ En Colombia se calcula que en 2000 se perdieron 974698 años de vida potencial por causa de la violencia intencional, ${ }^{10}$ la mayor parte en jóvenes.

A pesar de que las mujeres jóvenes muestran tasas de homicidios sensiblemente más bajas que los varones, sus vidas se afectan en grado considerable por la violencia de sus comunidades. ${ }^{11}$ La violencia juvenil no está restringida a la muerte $\mathrm{u}$ otras lesiones físicas, sino también a formas de daño psicológico, como el abuso emocional, verbal y la negligencia, además de la agresión sexual. ${ }^{12}$

De acuerdo con la OMS, en 2000 se perpetraron 199000 homicidios (una tasa de 9.2 por 100000 habitantes) en jóvenes de 10 a 29 años. Las tasas de homicidios varían en gran medida entre las diferentes regiones y países; la región de las Américas es la más violenta. ${ }^{13}$ Colombia, con una tasa de 84.4 por 100 000, y El Salvador, con una tasa de 50.2 por 100 000, figuran entre los países con las tasas más altas, de acuerdo con los registros de la OMS. En casi todos los países, las tasas son mucho mayores para varones respecto de las mujeres (una razón entre las tasas masculinas/femeninas de alrededor de 13), lo cual sugiere que ser varón es un factor de riesgo de carácter demográfico.

Entre 1985 y 1994, las tasas de homicidios de jóvenes se incrementaron en muchos países del mundo, en particular en el grupo de edad de 10 a 24 años. Es curioso que en Canadá, donde un tercio de los homicidios de jóvenes se comete con armas de fuego, las tasas se redujeron; en cambio, en Estados Unidos de América, donde 70\% de los homicidios se perpetra con armas de fuego, las tasas aumentaron. ${ }^{13}$

Las pandillas o maras son organizaciones mal definidas y juegan un papel importante en el proceso normal de los jóvenes para alcanzar autonomía. Estas agrupaciones son variables, desde un grupo inocuo de amigos que se congregan en una esquina hasta organizaciones altamente estructuradas que se dedican a actividades criminales. Es por esa razón que resulta difícil precisar la contribución de las pandillas a la violencia juvenil. En la ciudad de Cali, mediante un criterio relativamente estricto, se calculó que cerca de 10\% de los homicidios se debía a pandillas. ${ }^{14}$

Dado que no existe una definición precisa, es difícil valorar la magnitud del problema de las pandillas. En Estados Unidos de América se calculaba que en 1995 había alrededor de 25000 pandillas con un total de 650000 integrantes, de los cuales $54.6 \%$ tenía un origen africano y $32.6 \%$ un rasgo latino. ${ }^{15}$ Algunas pandillas deportadas de esa nación conservan el nombre original al llegar a su país de origen, como es el caso de las famosas y violentas Salvatrucha o la Dieciocho Calle en El Salvador. ${ }^{16}$

Según el Caribean Adolescente Health Survey de 1997, uno de cada 11 adolescentes informó que pertenecía a una pandilla o mara y $10 \%$ refirió una pertenencia pasada. Entre los jóvenes de 16 a 18 años, uno de cada nueve reconoció su intervención en una pelea con armas en el año anterior. ${ }^{17}$

\section{Prevención de la violencia y salud pública}

La violencia, que se consideró de manera inicial como del dominio exclusivo de la policía y la justicia criminal, convoca ahora a los científicos sociales, politólogos y expertos de salud pública. Tanto el Banco Interamericano de Desarrollo como el Banco Mundial consideran la violencia y la inseguridad como uno de los mayores obstáculos para el desarrollo. ${ }^{18,19}$

Desde que Abad Gómez propuso aplicar los métodos epidemiológicos para la violencia colombiana, ${ }^{20}$ 
la salud pública se ha interesado en la prevención de la violencia en las Américas. ${ }^{21}$ Los conceptos básicos tales como necesidad de información confiable, identificación de factores de riesgo, multicausalidad, complejidad de la red causal, junto con su aproximación empírica al problema, se aplicaron en la ciudad de Cali y luego en otras regiones de Colombia, con gran utilidad. ${ }^{22,23}$

\section{Criterios científicos para medidas funcionales}

Es común que los trabajadores de campo y activistas contra la violencia consideren innecesario emplear tiempo y recursos en estudios voluminosos para demostrar la utilidad de una intervención, evidente para ellos; les resulta suficiente la llamada "evidencia anecdótica", resultante del hallazgo de algunos resultados satisfactorios. Sin embargo, existen múltiples casos de intervenciones que se adoptaron con base en el éxito de casos aislados, pero cuya evaluación no muestra ningún valor. ${ }^{24}$

Por razones prácticas, la presente revisión sólo busca identificar aquellas experiencias o políticas que han mostrado de manera científica ser eficaces para el control de la violencia juvenil. El criterio es la efectividad contra la violencia juvenil, aunque muchas medidas son también eficaces para otras formas de violencia. No se incluyen en esta revisión experiencias que, si bien pueden parecer promisorias, no se han evaluado en grado suficiente.

La credibilidad de un estudio, desde el punto de vista científico, exige tres requisitos esenciales: en primer lugar, un diseño experimental riguroso; en segundo, evidencia estadística de un resultado satisfactorio; y, en tercero, resultados reproducibles, observados en otros lugares y por otros investigadores. ${ }^{25}$

En sentido estricto, un diseño riguroso para determinar la causalidad exige necesariamente la participación de grupos de intervención y grupos control, escogidos de manera aleatoria. De esta manera es posible evitar el llamado sesgo de asignación o selección, que hace que los grupos sean diferentes.

En un diseño experimental riguroso es también recomendable que los observadores de los resultados desconozcan el grupo al que pertenecen los sujetos, de tal modo que se controle lo que se conoce como el sesgo del observador. Cuando se logran controlar los dos sesgos, el diseño se conoce con el nombre de "doble ciego", un diseño de máximo poder probatorio. ${ }^{26}$

Con frecuencia, el investigador se ve en la necesidad de comparar a grupos similares, pero no seleccionados de manera aleatoria. Puesto que siempre existe la posibilidad de que algún factor desconocido, presente en alguno de los grupos, explique los hallazgos, este diseño tiene menor credibilidad que el anterior.

La evidencia estadística de un resultado satisfactorio (la denominada significancia estadística) indica que es poco probable que los resultados observados se deban al azar. El nivel de probabilidad escogido depende de las preferencias del investigador, con base en la importancia de equivocarse. La significancia estadística depende, además de la magnitud del efecto, del tamaño de la muestra. Un efecto pequeño encontrado en una muestra grande es poco probable que se deba al azar $y$, de manera contraria, un efecto grande puede pasar como explicado por la suerte -sin significado estadístico- si la muestra es pequeña.

En virtud de la estrecha correlación y superposición que se observan entre muchos de los factores de riesgo para la violencia juvenil (como deserción escolar temprana, desempleo y falta de ingresos, conductas sexuales de riesgo, consumo de alcohol y otras sustancias, etcétera), determinar la importancia de un factor de riesgo aislado o una política específica es un problema metodológico de gran envergadura, casi insoluble.

Al tratarse de prevención de la violencia juvenil, los resultados deben mostrar que las intervenciones la reducen. No obstante, en un caso supuesto, si las intervenciones ocurren a temprana edad (algunas se describen más adelante) el periodo de observación requerido es de varios años, ya que antes de la adolescencia no se pueden identificar. Para evitar este problema se acepta que una intervención es eficaz si altera un factor de riesgo comprobado. Por ejemplo, cuando un programa es eficaz para reducir el castigo físico del niño, factor de riesgo comprobado de violencia juvenil, se puede admitir que dicho programa es eficiente contra la violencia juvenil.

A continuación se presenta un resumen de las intervenciones o programas que se han probado, de acuerdo con los criterios ya mencionados. En algunos casos, el resultado ha sido la reducción del comportamiento violento y en otros la reducción de un factor de riesgo reconocido.

Es preciso señalar que aun las mejores intervenciones no pueden tener el éxito esperado si no se instituyen de manera correcta. Son necesarios personal entrenado y una supervisión adecuada para asegurar una fiel aplicación del modelo, además de contar con los recursos suficientes para aplicarlas por el tiempo mínimo estipulado.

\section{Niveles de prevención}

Un modelo muy utilizado en salud ${ }^{27}$ establece tres niveles de prevención. La prevención primaria hace referencia a las intervenciones que se aplican en la po- 
blación general, sin tener en cuenta su nivel de riesgo. Es el caso de las campañas para desarmar a la población general. La prevención secundaria alude a las intervenciones dirigidas a niños o jóvenes de riego elevado de violencia, incluso si no han cometido ningún crimen. La reducción de la violencia escolar, o bullying, puede considerarse como una intervención de esta categoría. La prevención terciaria se refiere a las medidas dirigidas a quienes ya han participado en actividades delictivas. La rehabilitación de prisioneros en las cárceles es una intervención típica de este nivel de prevención.

Como se verá más adelante, algunas intervenciones, como el entrenamiento en prácticas de crianza, pueden considerarse como prevención primaria o secundaria, según sea la población en que se apliquen.

Dadas las dificultades de seguimiento mencionadas con anterioridad, la mayor parte de los estudios se limita a mostrar la reducción de los factores de riesgo. En estos estudios, los factores de riesgo (o factores de protección) se identifican en edades tempranas y se comparan con el comportamiento violento en etapas posteriores.

Si alguna característica, ya sea personal, ya del medio ambiente, se vincula en grado positivo con comportamientos violentos se considera de riesgo y, en caso contrario, como factor protector.

La presencia de un factor de riesgo o protector indica tan sólo una probabilidad elevada o reducida en un grupo poblacional; no especifica con certeza que un niño que lo tenga será necesariamente violento. Es pertinente anotar que algunos factores de riesgo para violencia juvenil no son modificables, por ejemplo, ser varón.

\section{Intervenciones efectivas}

\section{Prevención primaria}

Las armas de fuego contribuyen de gran manera a la violencia homicida, en especial en los jóvenes. En Estados Unidos de América, 80\% de los homicidios juveniles se comete con armas de fuego.$^{28}$ En Colombia, de igual modo, las armas de fuego representan $80 \%$ de los homicidios totales. ${ }^{29} \mathrm{~A}$ la abundante presencia de armas de fuego en manos de la población general y las pandillas o maras se atribuye la persistente elevación de los tasas de homicidios en El Salvador, aun después de la firma de los acuerdos de paz. ${ }^{30}$

Un ejemplo de prevención primaria exitosa lo constituyen los esfuerzos para desarmar a la población civil en ciertos periodos, como los fines de semana o ciertas ocasiones especiales, que se han llevado a cabo en las ciudades de Cali y Bogotá, en Colombia. En estas poblaciones se observó una reducción significante de 14\% de los homicidios. ${ }^{31}$

\section{Prevención secundaria}

El desarrollo de las habilidades que más tarde en la vida influyen sobre el comportamiento emocional se determina en gran proporción por los canales neuronales desarrollados en los primeros años de vida. Por esta razón, los tres primeros años, cuando ocurre la mayor parte del desarrollo cerebral, son fundamentales, ya que la estimulación permite el adecuado desarrollo de estos tractos nerviosos. Experimentar violencia o abuso infantil, o ser testigo de violencia familiar, son factores de riesgo que influyen en la violencia juvenil. ${ }^{32}$

Otros factores de riesgo demostrado para la violencia juvenil son la participación de los padres en actividades criminales violentas, consumo de alcohol y otras drogas, maltrato infantil y negligencia. ${ }^{33}$ De igual manera, se conocen otros factores de riesgo, por ejemplo, las prácticas educativas violentas, la inconsistencia en la aplicación de normas disciplinarias, la falta de interacción entre padres e hijos o la nula supervisión. . $^{34,35}$

El desarrollo de programas dirigidos al desarrollo infantil temprano y las prácticas educativas adecuadas de los padres se consideran como algunas de las intervenciones de mayor beneficio respecto del costo para la reducción de las conductas juveniles de riesgo. ${ }^{36}$ Cuanto más temprano se inicien las intervenciones, más efectivas parecen. Las intervenciones, cuando los niños son todavía muy pequeños, son más efectivas respecto de cuando ya han desarrollado conductas agresivas.

Los padres pueden aprender métodos efectivos para la atención apropiada de sus hijos, pero un curso breve no es suficiente. Con frecuencia es necesario mantener una relación educativa con ellos, que puede extenderse desde algunos meses hasta varios años. Algunos programas de entrenamiento parental han demostrado su eficacia. ${ }^{37,38}$

Algunos de los principios de las intervenciones apropiadas son los siguientes: jugar con el niño en contraposición a dirigirlo; estimular y reconocer las conductas correctas; promover un refuerzo positivo acompañado de consistencia en las prácticas disciplinarias; y supervisar de manera permanente. ${ }^{39}$

Un estudio mostró que las madres pobres, cabezas de hogar, enfrentadas a muchos factores de estrés, tenían prácticas de crianza que desembocaban en conductas violentas de sus hijos. ${ }^{40}$ Otro estudio, con selección aleatoria de grupos, mostró que el entrenamiento del padre y la madre era beneficioso para mejorar el caso de los niños con problemas de comportamiento. ${ }^{41}$

Un estudio controlado en un número cercano a 
1000 estudiantes, realizado en ocho ciudades de Estados Unidos de América, demostró que la presencia de adultos voluntarios que dedicaban tiempo a interactuar con niños y adolescentes reducía hasta $46 \%$ el consumo de drogas, $32 \%$ las conductas agresivas violentas y $52 \%$ el ausentismo escolar. ${ }^{42}$

Algunos programas orientados al tiempo libre después de la escuela (tiempo durante el cual se eleva el riesgo de incurrir en conductas criminales) han mostrado efectos notorios en la reducción de la criminalidad juvenil, consumo de drogas y vandalismo. ${ }^{43,44}$

David P. Weikart desarrolló el llamado High/Scope Perry Preschool Program, uno de los pocos estudios que han tenido un diseño adecuado y un seguimiento suficiente para demostrar los efectos en el largo plazo. El proyecto incluyó a niños de alto riesgo, afectados por varios factores de riesgo (personales, ambientales y escolares) y aplicó medidas educativas activas que suponían la intervención de muchas personas; se estimulaban las conductas responsables independientes en los estudiantes, con énfasis en el desarrollo de habilidades del lenguaje, matemáticas, música y pensamiento lógico. Al compararlos con un grupo control, a la edad de 27 años, Weikart encontró que los graduados del programa habían tenido $68 \%$ menos detenciones por consumo de drogas y había $63 \%$ menos muchachos que podían considerarse criminales por haber sufrido más de cinco detenciones por la policía. En cuanto a los indicadores positivos, los egresados del programa tenían casa en una proporción del doble y $31 \%$ se había graduado de la secundaria. ${ }^{45}$

Un estudio que buscaba modificar las actitudes y el comportamiento hacia el castigo físico infantil mostró cambios significativos en las actitudes (reducción de 6\% en la aceptación del castigo físico) y el comportamiento (disminución de 10\% de la conducta de golpear con un instrumento duro) como herramientas educativas. El estudio se llevó a cabo en tres ciudades colombianas y contó con un diseño riguroso (con grupos de control aleatorios) e incluyó educación sobre los efectos dañinos del castigo físico; asimismo, tuvo un intenso trabajo comunitario y se sustentó en un programa de difusión y educación a través de medios masivos de comunicación nacionales. ${ }^{46}$

Irving y colaboradores ${ }^{47}$ mostraron la eficacia de la enseñanza de habilidades de crianza a padres de niños mayores en la disminución del riesgo de consumir drogas, problemas escolares y conducta antisocial. El programa, llamado Adolescent Transition Program, consistió en clases semanales para enseñar a los padres a escuchar de forma activa, utilizar estímulos, establecer reglas y acordar sanciones en caso de incumplimiento de ellas. Este estudio, que también incluyó una selección aleatoria de grupos, tuvo un seguimiento de cuatro años.

Mención especial merece el tema de la prevención del acoso escolar, también llamado matoneo o, por su expresión inglesa, bullying. El acoso consiste en humillar a un compañero, exponerlo a actos de crueldad física y emocional de manera permanente, por parte de uno o varios de sus pares. Su ocurrencia es tan frecuente que algunos lo consideran una etapa inevitable del crecimiento. Sin embargo, el daño que produce en las víctimas es muy grande y los victimarios tienen una mayor probabilidad de tener problemas con la ley, si no se los corrige de modo apropiado. Olweus desarrolló un programa, aprobado en 42 escuelas primarias y secundarias de Noruega, que identificó una reducción de $50 \%$ de los problemas de acoso escolar y una notable disminución del vandalismo y ausentismo escolar. ${ }^{48} \mathrm{El}$ programa se basa en el principio de aprender a distinguir el abuso escolar de las peleas y los conflictos de la vida diaria, reprimirlos cuando ocurren, sancionar a los agresores, conferir poder a las víctimas, incorporar a los estudiantes pasivos en la creación de una cultura escolar antiabuso y trabajar con los padres de los agresores para corregirlos y con los de las víctimas para fortalecerlos.

\section{Prevención terciaria}

Ciertos estudios analizan intervenciones exitosas con padres de niños mayores que ya incurrieron en conductas antisociales o abiertamente delictivas. Se trata de hogares uniparentales, de bajos ingresos, con antecedentes de abuso infantil, en los que se han aplicado prácticas inadecuadas de disciplina, con escasa interacción entre los padres y los hijos. En muchos de estos casos se desarrolla lo que Often ${ }^{39}$ llama "patrones destructivos de interacción coercitiva". Estos patrones se distinguen por una relación entre padres e hijos caracterizada por ciclos de comportamiento antisociales que los padres controlan con amenazas. Aunque efectivos a corto plazo, se ha advertido que se acompañan por ciclos posteriores de mayor agresión.

Patterson, Reid y Dishion desarrollaron una intervención dirigida a romper el ciclo de interacción coercitiva. ${ }^{40}$ La intervención incluye 12 reuniones semanales en las cuales se enseña a los padres, en grupo y en sesiones familiares individuales, entre otras cosas, habilidades como el seguimiento de los hijos, el estímulo para efectuar conductas proactivas, disciplina sin agresión y ejercicios para la solución de problemas. La evaluación mostró una mejoría de la unidad familiar y una mayor efectividad para la solución de problemas familiares.

Uno de los estudios que ha mostrado mayor beneficio en relación con el costo es la llamada terapia multisistémica de Henggeler, ${ }^{49}$ que se basa en la premisa según 
la cual la familia es parte de una red interconectada en la que padres e hijos interactúan con factores externos como la escuela y el ambiente laboral. Por lo tanto, las intervenciones dirigidas sólo a los padres pueden no ser eficaces si no hay cambios en el contexto en que éstos se desenvuelven. Un estudio, con grupos elegidos de manera aleatoria, en el cual se aplicó la terapia multisistémica a jóvenes que habían tenido al menos una conducta violenta, mostró que después de 59 semanas de seguimiento el grupo tratado tuvo menos detenciones que los jóvenes sometidos al tratamiento habitual. ${ }^{39}$

En el cuadro I se resumen algunos de los factores de riesgo o protección más conocidos, con base en el ambiente en que se aplican: individual, familiar, escolar, grupal y comunitario.

\section{Referencias}

I. Génesis 4:8.

2. Koop CE. Injury prevention: meeting the challenge. En:A Report of the National Committee for Injury Prevention and Control. Oxford: Oxford University Press, 1989:192-203.

3. Koop CE, Lundeberg GD.Violence in America: a public health emergency: time to bite the bullet back. JAMA 1992;267:3075-3076. 4. Pan American Health Organization. Resolution XIX, approved by the XXXVII Meeting of the Directing Council.Washington DC: PAHO, 1993. 5. World Health Organization. Resolution WHA49.25, approved by the Forty-ninth World Health Assembly. Geneva:WHO, 1996.

6. Ratinoff $L$, ed. Toward an integrated approach to development: ethics, violence and citizen safety. Report of colloquium held at the Interamerican Development Bank, February 16-17, 1996, Washington DC; The World Bank. Crime and Violence as development issues in Latin America and the Caribbean, presented at the Seminar The Challenge of Urban Criminal Violence, held by the Interamerican Development Bank,

\section{Cuadro I}

\section{FActores PRotectores y de RIESGo PARA LA VIOLENCIA EN EDADES dE I5 A I8 AÑos*}

\begin{tabular}{|c|c|c|c|}
\hline \multirow[b]{2}{*}{ Ámbito } & \multicolumn{2}{|c|}{ Factor de riesgo } & \multirow[t]{2}{*}{ Factor protector } \\
\hline & $\begin{array}{l}\text { Comienzo temprano } \\
\text { (edad, 6-II años) }\end{array}$ & $\begin{array}{l}\text { Comienzo tardío } \\
\text { (edad, I2-14 años) }\end{array}$ & \\
\hline Individual & $\begin{array}{l}\text { Infracciones varias } \\
\text { Ser varón } \\
\text { Consumo de alcohol y otras drogas } \\
\text { Conducta agresiva } \\
\text { Problemas psicológicos } \\
\text { Hiperactividad } \\
\text { Actitudes y conducta antisociales } \\
\text { Exposición a violencia en medios } \\
\text { Bajo Cl } \\
\text { Deshonestidad } \ddagger\end{array}$ & $\begin{array}{l}\text { Infracciones varias } \\
\text { Ser varón } \\
\text { Problemas psicológicos } \\
\text { Inquietud } \\
\text { Dificultad para concentrarse }{ }^{\ddagger} \\
\text { Conductas arriesgadas } \\
\text { Agresiones }{ }^{\ddagger} \\
\text { Actitudes y conducta antisociales } \\
\text { Crímenes contra personas } \\
\text { Bajo Cl } \\
\text { Consumo de alcohol y otras drogas }\end{array}$ & $\begin{array}{l}\text { Intolerancia a apartarse de las normas } \\
\text { Alto } \mathrm{Cl} \\
\text { Ser mujer } \\
\text { Orientación social positiva } \\
\text { Percepción de sanciones para transgresores }\end{array}$ \\
\hline Familiar & $\begin{array}{l}\text { Bajo nivel SE/pobreza } \\
\text { Padres antisociales } \\
\text { Mala relación padres-hijos } \\
\text { Disciplina muy rígida, laxa o inconsiste } \\
\text { Hogar con conflicto, desintegrado } \\
\text { Separación de los padres } \\
\text { Padres abusadores } \\
\text { Negligencia }\end{array}$ & $\begin{array}{l}\text { Mala relación padres-hijos } \\
\text { Disciplina muy rígida, laxa o inconsistente } \\
\text { Mala supervisión } \\
\text { Pobre compromiso de los padres } \\
\text { Padres abusadores, antisociales } \\
\text { Hogar con conflicto, desintegrado } \\
\text { Bajo nivel SE/pobreza }\end{array}$ & $\begin{array}{l}\text { Relación cálida y apoyo con padres u otros adultos } \\
\text { Aprobación paterna de compañeros } \\
\text { Adecuada supervisión parental } \\
\text { Tutoría de un adulto }\end{array}$ \\
\hline Escolar & $\begin{array}{l}\text { Actitud negativa } \\
\text { Mal desempeño }\end{array}$ & $\begin{array}{l}\text { Actitud negativa } \\
\text { Mal desempeño } \\
\text { Pérdida de grado }\end{array}$ & $\begin{array}{l}\text { Interés en la escuela } \\
\text { Reconocimiento por participar en actividades }\end{array}$ \\
\hline Grupal & $\begin{array}{l}\text { Lazos débiles } \\
\text { Compañeros antisociales }\end{array}$ & $\begin{array}{l}\text { Lazos débiles } \\
\text { Compañeros antisociales o delincuentes } \\
\text { Pertenencia a pandillas }\end{array}$ & Compañeros en actividades convencionales \\
\hline Comunitario & & $\begin{array}{l}\text { Crimen, drogas en vecindario } \\
\text { Desintegración social en vecindario }\end{array}$ & \\
\hline $\begin{array}{l}\text { * Adaptado d } \\
2001 \\
\text { ‡ Sólo para va }\end{array}$ & $\begin{array}{l}\text { e US Department of Health and Huma } \\
\text { arones }\end{array}$ & ,200I.Youth violence: A report of the su & on general.Washington DC: US Public Health Service, \\
\hline
\end{tabular}


Rio de Janeiro March 2-4, 1997.

7. Pan American Health Organization. Health conditions in the Americas. Scientific Publication 552. Washington DC: PAHO, 1994.

8. Yunes J, Zubarew T. Mortality from violent causes among adolescents and young people: a challenge for the Region of the Americas. Washington DC: Pan American Health Organization, 1997.

9. Pan American Health Organization. Adolescent program health situation analysis. Mortality database from Technical Health Information System. Washington DC: PAHO, 1999.

10. Instituto Nacional de Medicina Legal y Ciencias Forenses de Colombia. Centro de Referencia Nacional sobre Violencia. Forensis 2000. Bogotá, Colombia: INML, 2000.

II. Moser C, van Bonkhorst B. Youth violence in Latin America and the Caribbean: costs, causes and interventions. Urban Peace Program Series. Washington DC:The World Bank, 1999.

12. Heise L.Violence against women: the hidden burden. Washington DC: The World Bank, 1994.

13. Krug EG, Dahlbergh LL, Mercy JA, Zwi A, Lozano R, eds. World Report on Violence and Health. Geneva:WHO, 2002.

14. Espitia VE. Muertes violentas en Cali. 1993-1996. Cali: Secretaría de Gobierno, Convivencia y Seguridad de Cali.Alcaldía de Santiago de Cali, 1997.

15. Spergel I, Curry D, Chance R, et al. Gang suppression and intervention: problem and response. Research summary. Washington DC: Office of Justice Programs. US Department of Justice, 1994.

16. Organización Panamericana de la Salud. Informe de un taller sobre violencia de los adolescentes y pandillas juveniles. Washington DC: OPS, 1997:22-25

17. Pan American Health Organization. Caribbean Adolescent Health Survey:Antigua, Dominica, Grenada, Jamaica, Barbados. Washington DC: PAHO, 1998.

I8. Londoño JL, Guerrero R.Violencia en América Latina: epidemiología y costos. En: Londoño JL, Gaviria A, Guerrero R, comp. Asalto al desarrollo. Violencia en América Latina. Washington DC: Banco Interamericano de Desarrollo, 2000.

19. The World Bank. Crime and violence as development issues in Latin America and the Caribbean, presentado en el seminario El reto de la Violencia Criminal Urbana, organizado por el Banco Interamericano de Desarrollo, Río de Janeiro marzo 2-4, 1997.

20.Abad-Gómez H. La violencia necesita estudios epidemiológicos. Tribuna Médica 1962;2:9-12.

2I. Foege WH, Rosenberg ML, Mercy JA. Public health and violence prevention. Curr Iss Publ Health 1995; I:2-9.

22. Guerrero R, Concha A, Álvarez A, Cobo G, De Roux G, Alzate A. Programa Desarrollo, Seguridad y Paz DESEPAZ. Estrategias de la Alcaldía de Cali para enfrentar la inseguridad y la violencia. En: Concha A, Carrión F, Cobo G, comp. Ciudad y violencias en América Latina. Quito: Programa de Gestión Urbana, 1994:1 I9-153.

23. Martin G, Ceballos M. Bogotá: anatomía de una transformación. Bogotá: Editorial Pontificia Universidad Javeriana, 2004:219-254.

24. Ringwalt C, Greene J, Ennett S, lacham R, Clayton RR, Leukefekd CG. Past and future directions of the DARE program: an evaluation review: Draft Final Report National.Washington DC: Institute of Justice, 1994. 25. US Department of Health and Human Services. Youth violence: a report of the surgeon general.Washington DC: US Public Health Service, 2001 .

26. Guerrero R, González C, Medina E. Epidemiología. Bogotá: Fondo Educativo Interamericano, I98I.

27. Devine J, Gilligan J, Miczek KA, Shaik R, Pfaff D, comp. Youth violence. Scientific Approaches to Prevention. New York:The New York Academy of Sciences, 2004.

28.The Carter Center. Not even one.The Report of the Carter Center
Consultation on the Crisis of Children and Firearms.Atlanta GA:The Carter Center, 1994.

29. Instituto Nacional de Medicina Legal y Ciencias Forenses. Reporte del comportamiento de las lesiones fatales en Colombia, 1994. Bogotá: Centro de Referencia Nacional sobre Violencia, 1994.

30. Organización Panamericana de la Salud. Informe de un taller sobre violencia de los adolescentes y pandillas juveniles. Washington DC: OPS, 1997:22-25

3I.Villaveces A. Effect of a band on carrying firearms on homicide rates in two Colombian cities. JAMA 2000;283(9): 1205-1210.

32. World Health Organization and International Society for Prevention of Child Abuse and Neglect. Preventing child maltreatment: a guide to taking action and generating evidence. Geneva:WHO, 2006.

33. US Department of Health and Human Services. Youth violence: a report of the surgeon general.Washington DC: US Public Health Service, 2001

34. Buka S, Earls F. Early determinants of delinquency and violence. Health Affaires 1993;46-64.

35. Tolan P, Guerra N.What works in reducing adolescent violence: an empirical review of the field. Boulder CO: Center for the Study and Prevention of Violence. University of Colorado, Boulder, 1994.

36. World Health Organization and International Society for Prevention of Child Abuse and Neglect. Preventing child maltreatment: a guide to taking action and generating evidence. Geneva:WHO, 2006.

37. Webster-Stratton C. Preventing conduct problems in head start children: strengthening parenting skills.J Consul Clin Psychol 1998;66: 715-730.

38. Kasdin A. Parent management training: evidence, outcomes and issues. J Am Acad Chil Adol Psy 1997;36:1349-1358.

39. Thornton TN, Craft CA, Dahlberg LL, Lynch BS, Baer K. Best practices of youth violence prevention: a sourcebook for community action. Atlanta: Centers for Disease Control and Prevention, National Center for Injury Prevention and Control, 2000.

40. Patterson GR, Reid JB, Dishion TJ. 1992. A social interactional approach IV: antisocial boys. Eugene OR. Castalia Publishing.

4I.Webster-Stratton C. Randomized trial of two parent-training programs for families with conduct-disordered children. J Consul Clin Psychol 1984;52(4):666-678.

42. Grossman JB, Gary EM. Mentoring. A proven delinquency prevention strategy. Washington DC: Juvenile Justice Bulletin Department of Justice, 1997.

43. Schinke SP, Orlando MA, Cole KC. Boys and girls clubs in public housing developments: prevention services for youth at risk. J Comm Psychol (OSAP Special Issue) 1992; I 18-128.

44. Jones MB, Oxford DR. Reduction of antisocial behavior in poor children by non school skill development. J Child Psychol Psy All Dis 1989;30:737-750.

45. The High/Scope Perry Preschool program. Disponible en: http: modelprograms.samhsa.gov.

46. Muñoz E, Gutiérrez Ml, Guerrero R. Evaluación del impacto de una estrategia de información, educación y comunicación para la prevención de la violencia. Cali, Colombia: Ministerio de la Protección Social de Colombia. Universidad del Valle, Instituto CISALVA, 2004.

47. Irving $A B$. The effectiveness of a parenting skills program for parents of middle school students in small communities. J Consul Clin Psychol 1999;67(6):8II-825.

48. Olweus D. Bully/victim problems in school: knowledge base and an effective intervention program. Irish J Psychol 1997;18:170-190. 49. Henggeler SW, Melton GB, Smith LA, Schoenwald SK, Hanley JH. Family preservation using multisystemic treatment: long term follow-up to a clinical trial with serous jevenile offenders. J Child Fam Stud 1993;2:283-289. 